\title{
An unusual cause of anaemia
}

\author{
Paul F Findlay, John Sutherland
}

A 76-year-old woman was admitted to hospital with a history of increasing shortness of breath, pallor, and tiredness. Prior to admission her general practitioner had checked a full blood count and she was found to be anaemic with a haemoglobin of $5.3 \mathrm{~g} / \mathrm{dl}$. There was no history of overt blood loss, change in bowel habit, or melaena stool. She had been taking aspirin, but there was no history of haemoptysis or epistaxis. She had a history of iron deficiency anaemia, for which no cause was established, a long history of sero-negative rheumatoid disease, and an above-knee amputation following a thrombotic occlusion of a right femero-popliteal bypass graft originally inserted for a severely ischaemic pre-gangrenous foot. On examination she was noted to be pale, with facial and lingual telangiectasia. There were features of rheumatoid disease affecting the hands but no evidence of scleroderma. Aside from fine late inspiratory bi-basal crackles the remainder of the examination was unremarkable.

Investigations confirmed an iron deficiency anaemia with a haemoglobin of $5.3 \mathrm{~g} / \mathrm{dl}$, mean corpuscular volume $67 \mathrm{fl}$, and ferritin $3.3 \mu \mathrm{g} / 1$ (normal range 10-350). Red cell folate, vitamin B12, and a clotting screen were all normal. A chest X-ray revealed bilateral interstitial changes in the lower zones. Following transfusion of four units of packed red cells the facial and lingual telangiectases became more noticeable (figure). ${ }^{1}$

Department of

Medicine for the

Elderly, Woodend

Hospital, Eday Road,

Aberdeen AB15 6LS,

UK

P F Findlay

J Sutherland

Accepted 10 March 1998

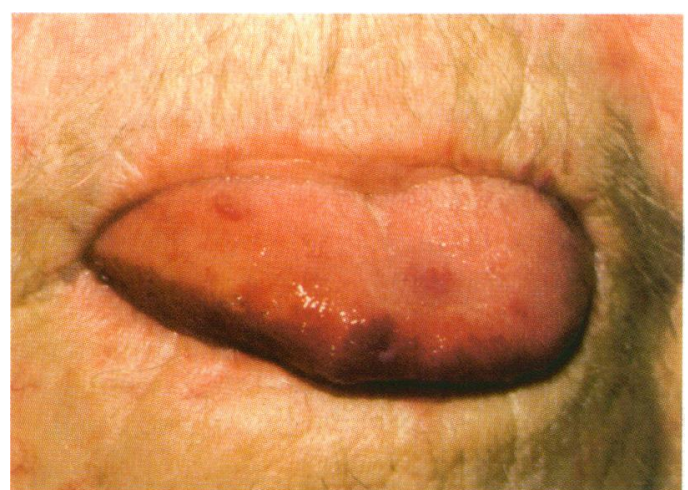

\section{Questions}

1 What is the probable diagnosis?

2 What complications can occur?

Figure 
Answers

QUESTION 1

Hereditary haemorrhagic telangiectasia (HHT) or Osler-Weber-Rendu disease. Upper gastrointestinal endoscopy confirmed the presence of telangiectatic lesions in the stomach and duodenum. There were also some superficial gastric mucosal erosions but no evidence of ulceration. It is quite typical for the lesions to become more obvious following blood transfusion.

QUESTION 2

The complications associated with HHT are listed in the box.

\section{Discussion}

HHT is an autosomal dominant fibrovascular dysplasia which results in telangiectasia, arteriovenous (AV) malformations, and local aneurysmal dilatation. The lesions may be visceral as well as mucocutaneous and the disorder may present with protean clinical manifestations depending on the sites involved. Typically there is a family history, although in $20 \%$ of cases this is not elicited and reflects incomplete penetrance of the disorder. Although epistaxis is a common presenting feature, particularly in younger age groups, the disorder may remain undetected for many years, as in this case. The cardinal gastrointestinal manifestation is painless bleeding from telangiectatic lesions in the upper and lower gastrointestinal tract. In addition, there is an increased incidence of duodenal ulceration in these patients. ${ }^{23}$ Involvement of the liver may result in hepatomegaly and right upper quadrant pain, a pulsatile mass or an audible bruit. These features are a consequence of increased hepatic blood flow via AV fistulae and can result in a hyperdynamic circulatory state and high output cardiac failure. Rarely, portosystemic shunting results in encephalopathy after a gastrointestinal bleed.

HHT is the commonest disorder associated with pulmonary AV fistulae in all age ranges. Approximately $15 \%$ of patients with overt

1 Swain DG. Profound anaemia in an elderly man. Postgrad Med f 1996;72:369-70.

2 Reilly PJ, Nostrant TT. Clinical manifestations of hereditary haemorrhagic telangiectasia. Am $\mathcal{F}$ Gastroenterol 1984;79: 363-7.

\section{Complications of HHT}

- iron deficiency anaemia

- epistaxis

- painless gastrointestinal bleeding

- duodenal ulceration

- hepatic AV fistulae

- pulmonary AV fistulae

- paradoxical emboli

- neurological complications

HHT have pulmonary fistulae and $60 \%$ have this feature in isolation. ${ }^{4}$ Blood from the pulmonary artery is shunted via these fistulae and can result in central cyanosis, hypoxaemia and secondary polycythaemia. Clinically bruits may be present on auscultation, particularly over the lower lobes. An important consequence of shunting is paradoxical embolism. Peripheral microemboli are at liberty to bypass the pulmonary capillary filter and such emboli, which may be septic, have a prediliction for the cerebral circulation.

Neurological complications can occur in all age groups but peak incidence is noted in the third to fourth decades. Paradoxical emboli and also intracranial vascular anomalies, resulting in intracerebral and subarachnoid haemorrhage, account for the majority. HHT can be a rare cause of stroke in a young person. Despite the complications, longevity is not significantly reduced nor is the quality of life greatly impaired. Fewer than $10 \%$ die as a result of complications relating to the disorder. Iron and folate supplements are required, and aspirin and other treatments that impair haemostasis are contraindicated.

\section{Final diagnosis}

Hereditary haemorrhagic telangiectasia.

Keywords: anaemia; hereditary haemorrhagic telangiectasia

3 Smith CR, Bartholomew LG, Cain JC. Hereditary hemorrhagic telangiectasia and gastrointestinal hemmorhage. Gastroenterology 1963;44:1-6.

4 Dines DE, Arms RA, Bernatz PE, Gomes MR. Pulmonary arteriovenous fistulas. Mayo Clin Proc 1974;:49:460-5. 